\section{CONCURSO DE PREMIOS EUROPEOS DEL CEMBUREAU-1982}

$161-209$

\section{Sinopsis}

En este artículo se recogen los cinco primeros premios concedidos en el concurso organizado por el CEMBUREAU, en el año 1982, sobre «El hormigón en la vivienda unifamiliar y en los pequeños edificios de vivienda colectiva».

Además de las notas técnicas de cada uno de los trabajos premiados se adjunta el comentario del jurado para cada uno de ellos.

Con el fin de promocionar en la vivienda unifamiliar y en los pequeños edificios de vivienda colectiva el uso del hormigón, material que, a pesar de su economía de inversión y mantenimiento y de sus posibilidades arquitectónicas, es frecuentemente menospreciado y comúnmente no considerado en construcciones de este tipo, el Cembureau organizó un concurso a mediados de 1982, al que se presentaron 57 trabajos procedentes de doce países, de los cuales 32 correspondían a la $1 .^{\text {a }}$ categoría (viviendas unifamiliares) y los restantes a la $2 .^{a}$ (edificios colectivos pequeños).

En la 1. a categoria se concedieron premios a tres trabajos: dos primeros premios, compartidos, para un proyecto de viviendas desarrolladas a lo largo de una calle comercial y para una vivienda unifamiliar; y un tercero para un edificio de viviendas armonizado con el estilo de los edificios circundantes.

En la 2. ${ }^{\text {a }}$ categoría se otorgaron dos premios: el primero correspondió a un conjunto de viviendas que mantienen una afinidad con la trama urbana existente; y el segundo, a un edificio de apartamentos de concepción original.

\section{1. ${ }^{a}$ Categoria: Vivienda unifamiliar.}

\section{Desarrollo de calle comercial Perth, Reino Unido.}

Arq.: James Parr y asociados.

Ing.: Sir Murdo MacDonald y asociados.

\section{Primer premio compartido.}

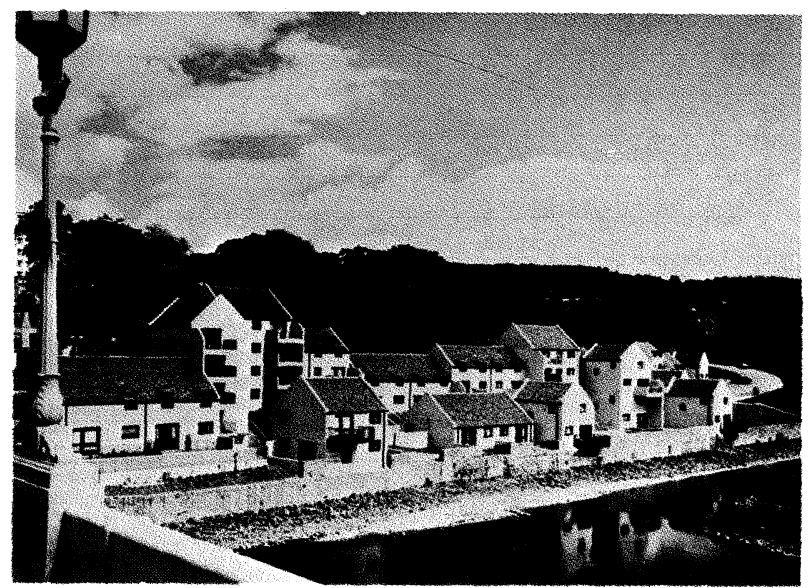

En una calle comercial, situada a la orilla del río, se ha realizado esta construcción que distribuye 40 viviendas unifamiliares de gran variedad de tipos y tamaños, oscilando desde la de dos personas hasta la de cinco, y construidas con materiales de calidad para que no desentonaran con la belleza del emplazamiento. Este último tuvo que ser remodelado enteramente para que, visto desde la otra orilla del río, se obtuvieron los mejores resultados posibles $y$, al mismo tiempo, para que se revalorizaran las perspectivas desde las casas a construir.

Con esta realización se ilustra el empleo de bloques de hormigón en la implantación de un nuevo conjunto de viviendas en el centro de una ciudad antigua, respetando la escala humana y acorde con ella.

En la construcción se emplearon bloques de hormigón, tratados al chorro de arena, de color rosado, para armonizar con la arenisca rojiza natural utilizada en el puente viejo contiguo a las obras y en otros edificios de las proximidades. Para conseguir un correcto aislamiento acústico en las paredes medianeras se utilizaron bloques macizos de hormigón, mientras que para el revestimiento interior de los muros exteriores se emplearon bloques más ligeros. 
Aunque los detalles de la anrica de bloques revisten una gran sencillez, el so de bloques especiales y su composición arquıtectónica confieren un gran interés a esta realización.

\section{Comentarios del jurado.}

La disposición general de estas modestas viviendas, acabadas con toda clase de cuidados y armoniosamente agrupadas, puede aportar también una contribución a una adecuada combinación de habitáculos que, al mismo tiempo, respeten y destaquen los emplazamientos naturales más privilegiados.

\section{* $\quad * \quad *$}

\section{Casa Ferrenbach}

Kaysersberg, Francia.

Arq.: Estudio de Urbanismo y Arquitectura UA5 Ing.: Oficina de Estructuras «K».

Decoración: Jacques Bertoux.

\section{Primer premio compartido.}

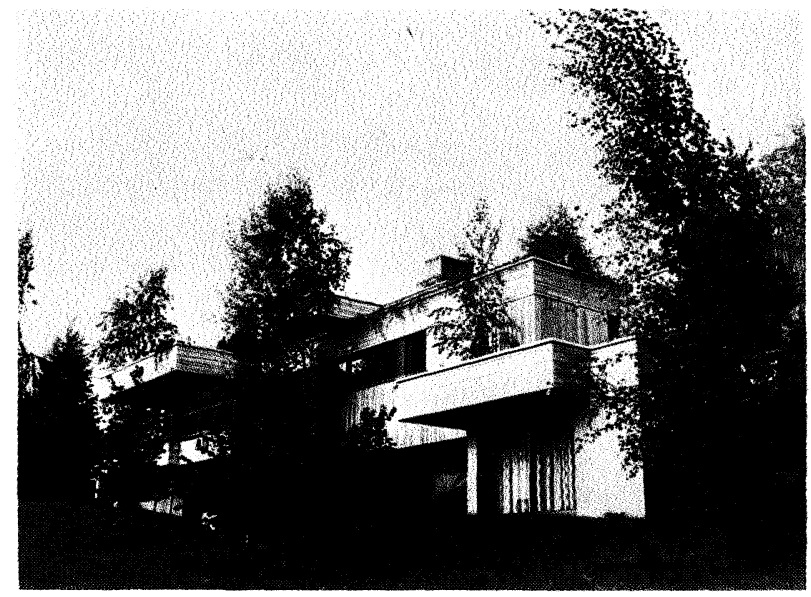

La composición elegida para esta vivienda unifamiliar, situada en el Valle de Saint Jean, cercano al centro de Kaysersberg, en los Vosges, surgió del completo análisis de una extensa gama de requisitos, de los que se dedujeron tres condicionantes esenciales: adaptación al emplazamiento de la mejor forma posible, proporcionar la mejor orientación a las distintas dependencias de la vivienda de acuerdo con su función, y conseguir una organización que posibilitara una forma de vida confortable $y$ acogedora.

En la realización de los cerramientos exteriores se emplearon exclusivamente dos materiales: el hormigón, dejado visto después de conformarlo con encofrados especiales, y la arenisca de Vosges, material tradicional en las construcciones de la región.
Estos mismos , nateriales se utilizaron también profusamente en el interior de la vivienda, a veces como prolongación de los muros exteriores y, otras, como elementos independientes. Así, en los muros de contención se empleó fábrica de hormigón de $30 \mathrm{~cm}$ de espesor revestida de mampostería. La estructura y los cerramientos exteriores se hicieron mediante una combinación de fábrica de hormigón armado de $16 \mathrm{~cm}$, aislamiento y doblado con tabique de ladrillo. En los muros no portantes se utilizó hormigón en masa, lavado, de 16 a $20 \mathrm{~cm}$ de grosor. También se utilizó el hormigón en los suelos, en este caso mediante losas macizas armadas de $16 \mathrm{~cm}$ de espesor mínimo, y solado de $6 \mathrm{~cm}$. Esta construcción maciza garantiza al mismo tiempo un correcto aislamiento acústico entre los diversos locales de la vivienda.

\section{Comentarios del jurado}

Esta casa, que hace un uso excelente del emplazamiento, causa un efecto bien proporcionado y equilibrado entre la marquesina horizontal y las nervaduras verticales, impresionando por la calidad del hormigón empleado.

$$
\text { * } * *
$$

\section{Viviendas unifamiliares en hilera.} Sódertull, Visby, Suecia.

Arq.: Bengt Agren/Ericson, Gynnerstedt \& Agren Ing.: Skanska Cementgjuteriet AB.

\section{Tercer premio.}

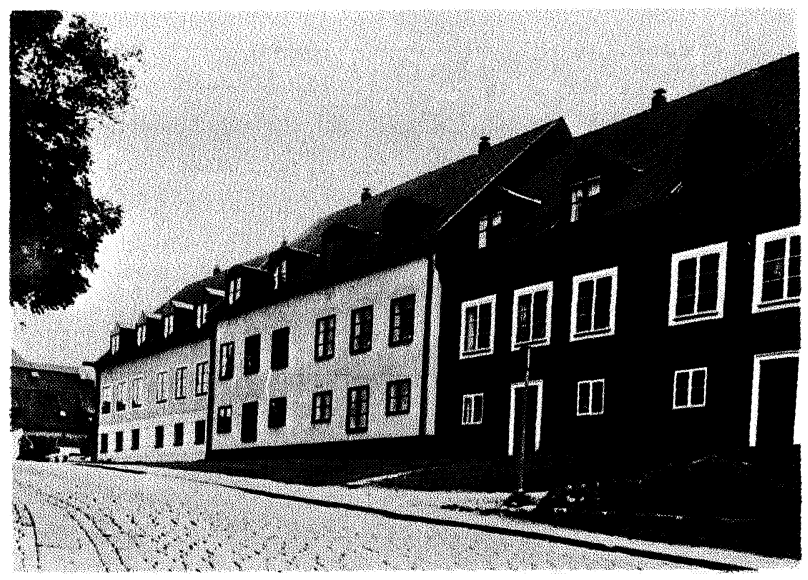

Este grupo de edificios está situado en las proximidades de una muralla medieval. El emplazamiento de las viviendas tiene una ubicación elevada, con una bella perspectiva de la población, del puerto y del mar Báltico. 
Informes de la Construcción/342

Desde la zona de aparcamiento se llega a un patio interior colectivo en el que se localizan todas las entradas de las casas. En dicho patio se encuentran también unas áreas de juegos al aire libre y las instalaciones de los servicios comunitarios.

Las viviendas son del tipo urbano tradicional en la zona, con dos plantas sobre rasante, más un nivel abuhardillado y un sótano. La superficie residencial por vivienda es de $125 \mathrm{~m}^{2}$, a los que se suman los $65 \mathrm{~m}^{2}$ del sótano.

El sistema constructivo empleado en la ejecución de estas viviendas se sale de lo normal; en esencia consiste en una envoltura estructural compuesta por unos elementos angulares prefabricados de hormigón, de $12 \mathrm{~cm}$ de espesor, enlazados entre sí con dobles fábricas también de hormigón. El mismo sistema se utiliza en los muros medianeros de las viviendas. Exteriormente los cerramientos se terminan con un revoco basto, combinado con otras zonas donde el acabado superficial es más fino.

\section{Comentario del jurado.}

Se trata de un ejemplo excelente de cómo las estructuras de hormigón modernas pueden participar en la renovación y crecimiento de comunidades establecidas, sin romper el estilo o uniformidad de los lugares interesados.

\section{* * * *}

2. ${ }^{a}$ Categoría: Pequeños edificios de vivienda colectiva.

\section{Viviendas colectivas en una comunidad residenciaí}

Marolles, Bélgica.

Arq.: Oficina de Arquitectura GUS

Ing.: Michel Dierckx.

\section{Primer premio.}

Una de las principales características de este conjunto habitacional es la doble orientación de las viviendas, que así pueden disfrutar del contacto con la animada vida de la calle por un lado, y de la tranquilidad, sosiego y carácter privado del patio interior ajardinado, por el otro.

También tuve gran importancia, en la concepción del proyecto, el interés por conectar directamente con el jardín el mayor número posible de viviendas, aunque fuera a través de escaleras que enlazaran

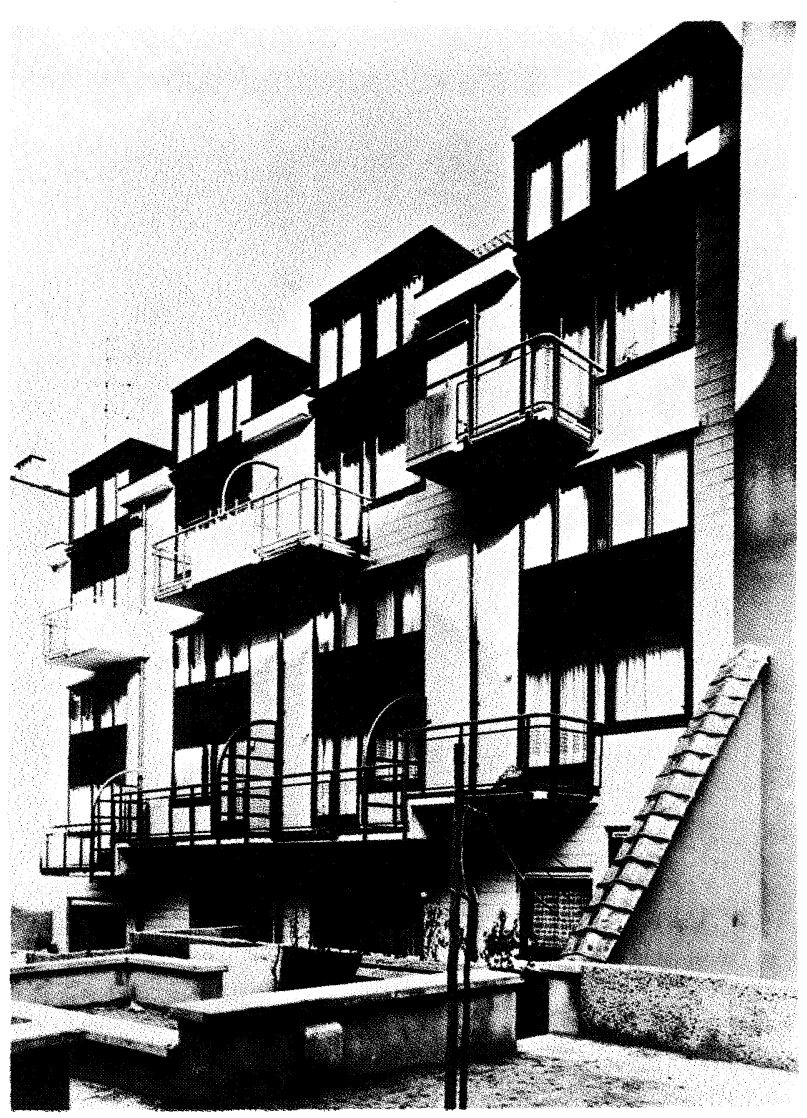

con las terrazas del primer nivel. Para ello era obligada la distribución de las viviendas en edificios de poca altura, y así, con esta disposición, se ha conseguido que seis de cada diez viviendas tengan acceso directo al jardín.

En la construcción del edificio se ha empleado una estructura de hormigón armado combinada con cerramientos portantes realizados con bloques de hormigón. Estos últimos se han acabado con pintura plástica texturizada para armonizar, en cuanto a dimensiones, aspecto y color, con los materiales empleados en las fachadas de la mayoría de los edificios existentes en la zona. En los suelos se han usado losetas lisas de hormigón en masa.

Para aislar acústicamente las viviendas de las cajas de escalera, éstas se han hecho con bloques macizos de hormigón, de $19 \mathrm{~cm}$ de espesor, y además se han separado de los demás elementos estructurales.

\section{Comentarios del jurado.}

Este edificio de apartamentos - buen ejemplo de renovación de la ciudad - demuestra que los bloques de hormigón pueden proporcionar un medio económico y atractivo para mantener una cierta unidad en la trama urbana.

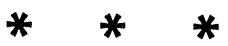




\section{Residencia de artistas}

Pescara, Italia.

Arq.: Francesco Berarducci y Donatella Ciocca Ing.: Carmine Barone.

\section{Segundo premio}

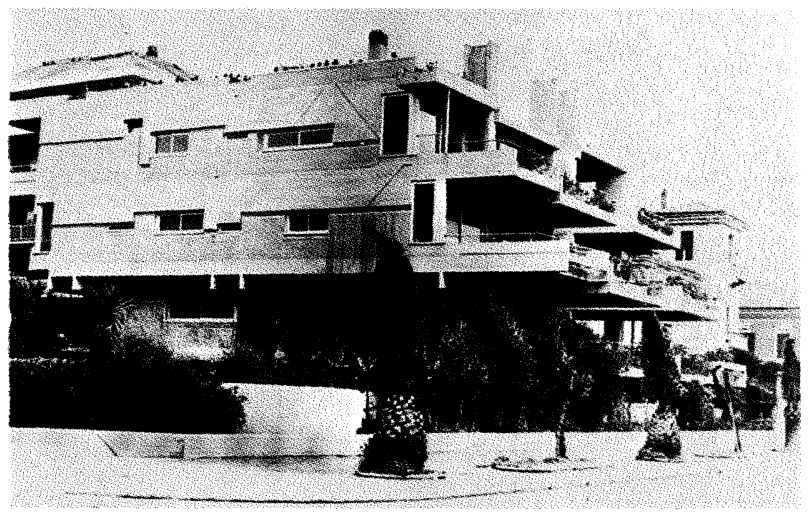

El edificio se encuentra ubicado en una zona muy centralizada situada a lo largo de la orilla del mar. Consta de dos partes: El volumen por encima de rasante está asignado a uso residencial, mientras que la parte enterrada la ocupará un auditorio para uso de los propios residentes.

La posibilidad de vistas panorámicas sobre el mar marcó unas prioridadés en cuanto a la situación de las distintas habitaciones de cada vivienda, y asi los salones y comedores se orientaron hacia esa perspectiva; los dormitorios y servicios se situaron en los laterales del edificio, y las cocinas en la parte trasera. Se consiguió aún más partido de dicha orientación por la ampliación de los salones mediante terrazas escalonadas.

La estructura está concentrada en unos pocos elementos, lo que posibilita una organización flexible en el sentido longitudinal. Para los forjados se han empleado vigas dobles colocadas ortogonalmente.

Los cerramientos exteriores, especialmente los de los costados, ya que los frontales los ocupan grandes ventanales, están realizados con muros de hormigón armado de $15 \mathrm{~cm}$ de espesor. Para su conformación se emplearon unos encofrados de diseño especial que configuran un marcado juego de volúmenes. El aislamiento, tanto térmico como acústico, se consigue por la interposición de una capa de aislante entre el muro exterior y el doblado interior con ladrillo de cara vista.

\section{Comentarios del jurado.}

Este grupo de apartamentos evidencia, por su concepción en terrazas, una aplicación satisfactoria de las posibilidades estructurales del hormigón, destacadas, en este caso, por una excelente ejecución.

\section{* * * *}

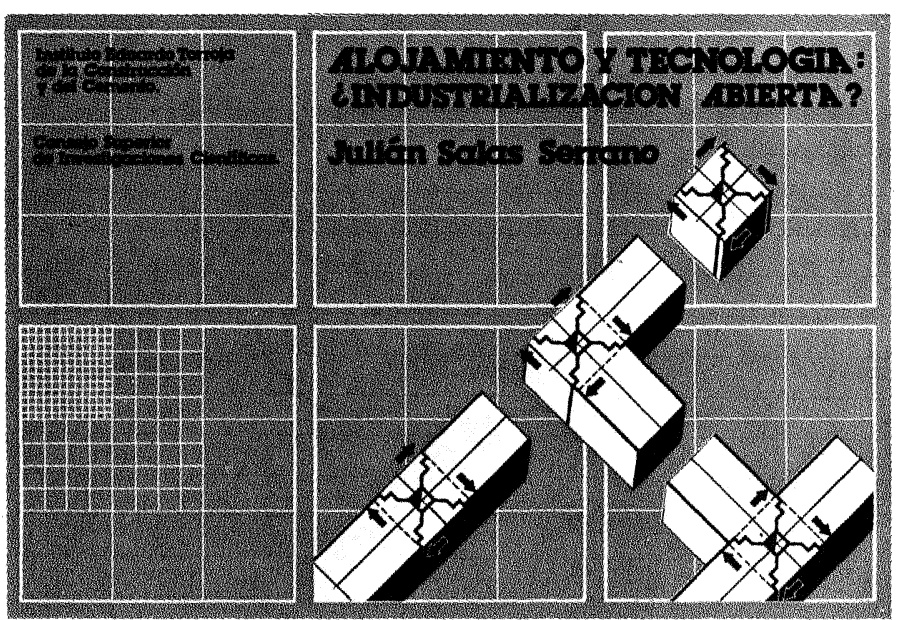

\section{ALOJAMIENTO Y TECNOLOGIA: ¿INDUSTRIALIZACION ABIERTA?}

JULIAN SALAS, ING. IND. (I.E.T.C.C.)

Un volumen de 160 páginas, 109 figuras y 16 tablas. Tamaño $240 \times 168 \mathrm{~mm}$. Encuadernado en rústica. Precios: España, 1.200 ptas; extranjero, 17 \$ USA.

\section{SUMARIO:}

Prólogo Prof. G. Ciribini.

\section{Introducción}

Capítulo 1.- La industrialización en las proclamas y ma nifiestos de arquitectura.

Capítulo 2.-¿Réquiem por la construcción industrializada?

Capítulo 3. - Algunos conceptos básicos.

Capítulo 4. - ¿Proyecto tradicional, construcción industrializada?

Capítulo 5.-Componentes.

Capitulo 6. - La coordinación dimensional hoy.

Capitulo 7.-Flexibilidad, intercambiabilidad y catálogos

Capítulo 8. - Industrialización, normativa y calidad.

Capitulo 9.-Reflexiones finales.

publicación del

INSTITUTO EDUARDO TORROJA 